\title{
Temporal and One-Step Stabilizability and Detectability of Time-Varying Discrete-Time Linear Systems
}

\author{
L. Gerard Van Willigenburg ${ }^{1, *}$ and Willem L. De Koning ${ }^{2}$ \\ ${ }^{1}$ Systems \& Control Group of Wageningen University, \\ P.O. Box 17, 6700 AA Wageningen, The Netherlands \\ gerard.vanwilligenburg@wur.nl \\ ${ }^{2}$ Department of Mathematics of Delft \\ University of Technology, Kroeskarper 6, Leiden, The Netherlands \\ wilros@planet.nl
}

\begin{abstract}
Time-varying discrete-time linear systems may be temporarily uncontrollable and unreconstructable. This is vital knowledge to both control engineers and system scientists. Describing and detecting the temporal loss of controllability and reconstructability requires considering discrete-time systems with variable dimensions and the $\mathrm{j}$-step, k-step Kalman decomposition. In this note for linear discrete-time systems with variable dimensions measures of temporal and one-step stabilizability and detectability are developed. These measures indicate to what extent the temporal loss of controllability and reconstructability may lead to temporal instability of the closed loop system when designing a static state or dynamic output feedback controller. The measures are calculated by solving specific linear quadratic cheap control problems.
\end{abstract}

Keywords: Temporal system properties, linear discrete-time systems, cheap LQ control problems, j-step k-step Kalman decomposition.

\section{Introduction}

Feedback control design and stability analysis of nonlinear systems along trajectories is often performed using the linearized dynamics about the trajectory [1], [2]. If the trajectory is time-varying the linearized model is time-varying. If in addition the nonlinear dynamics or the controls are non-smooth, i.e. in the case of bang-bang or digital control, the structure of the time-varying linearized system may change. Even if the nonlinear dynamics and the controls are smooth the structure of the timevarying linearized system may almost change. For control system design this is vital information since this structure reveals the temporal loss of controllability and reconstructability of the linearized system. They in turn may lead to temporal instability of a closed-loop control system [3], [4]. Recently we investigated these issues for continuous-time systems assuming continuous-time control. This investigation lead to the introduction of the properties temporal and differential

\footnotetext{
* Corresponding author.

D. Hömberg and F. Tröltzsch (Eds.): CSMO 2011, IFIP AICT 391, pp. 306-317,2013.

(C) IFIP International Federation for Information Processing 2013
} 
stabilizability and detectability for continuous-time linear systems [5]. In addition measures of these properties were introduced and calculated by solving specific linear quadratic cheap control problems [5], [6], [7].

Associated with computer control are digital control problems (sampled-data control problems). They concern the control of continuous-time systems by means of piecewise constant controls using sampled measurements. A common approach is to transform such control problems into equivalent discrete-time control problems [8], [9], [10]. Following this approach feedback control system design is performed in discrete-time. This motivates the discrete-time development in this paper that on the one hand parallels, but on the other is also very different from the one in continuous-time. The fact that discrete-time is not dense, as opposed to continuous-time, causes some major differences. In continuous-time our investigation required the introduction of piecewise constant rank systems and the differential Kalman decomposition [3], [4]. In discretetime their counterparts are discrete-time linear systems with variable state dimensions and the j-step, k-step Kalman decomposition [11].

This paper develops measures of temporal stability of time-varying linear discretetime systems over arbitrary finite time intervals, notably intervals where controllability or reconstructability is lost temporarily. Associated to this, measures of temporal and one-step stabilizability and detectability are developed. These measures can for instance be used to analyse temporal instability of a closed loop control system design using LQG output feedback.

Temporal stability may sound as a contradiction because formally stability relates to behavior when time tends to infinity. However, in one of his early seminal papers [12] Kalman together with Bertram already proposed measures of stability over finite time intervals (page 386). Intuitively stability relates to growth of the system state. Intuitively over intervals where the state grows we call the system temporal unstable and over intervals where the state decays, we call the system temporal stable. This intuition is formalized by the temporal stability property proposed in this note. This property is derived from a measure of temporal stability also proposed in this note that measures the maximum growth of the state over an arbitrary interval. Our concept of stability over a finite time interval differs from what is called finite-time stability [13], [14]. The reason we make a different choice is that our measures, their computation and the associated control system designs, come down to solving standard LQ problems. The standard LQ problems are of a special type called cheap control LQ problems [6], [7] . They are characterized by a control penalty that tends to zero. Computations and control system design associated to finite-time stability concern matrix inequalities [13], [14]. Generally these are much more difficult to solve.

\section{Temporal and One-Step Stability, Stabilizability and Detectability}

Temporal uncontrollability/unreachability and temporal unreconstructability/ unobservability of linear time-varying systems was introduced and investigated in continuous-time [3], [4] and in discrete-time [11]. Intuitively, temporal stabilizability 
and temporal detectability are associated properties that apply over intervals where the system is temporal uncontrollable/unreachable and temporal unreconstructable/unobservable respectively. In continuous-time this was formalized in [5]. In this section we formalize the discrete-time case. This requires considering variable dimension discrete-time linear systems (VDD systems) [11], [15], [16], [17] as well as $\mathrm{j}$-step controllability, $\mathrm{j}$-step reachability, k-step reconstructability, k-step observability and the associated j-step, k-step Kalman decomposition. All these are introduced in [11] that relies partly on [18]. In this section we consider VDD systems with a time domain $\left[i_{0}, i_{N}\right]$ where $i_{0}$ may tend to $-\infty$ and $i_{N}$ may tend to $+\infty$. Intervals where the VDD system is temporal uncontrollable/unreachable or temporal unreconstructable/unobservable are denoted by $\left[i_{s}, i_{f}\right]$.

\section{Definition 1.}

A VVD system is called $j$-step unreachable over the interval $\left[i_{s}, i_{f}+j\right] / j$-step uncontrollable over the interval $\left[i_{s}-j, i_{f}\right], \quad i_{0}+j \leq i_{s}<i_{f} \leq i_{N}-j \quad$ if $\forall i \in\left[i_{s}, i_{f}+j\right]$ the system is not j-step reachable at time $i$ / not j-step controllable from time $i-j$.

\section{Lemma 1.}

If $i_{s}, i_{f}$ satisfy the conditions in Definition 1 then over the interval $\left[i_{s}, i_{f}\right]$ the VDD system is 1) not $\mathrm{j}$-step reachable at each time and 2) not $\mathrm{j}$-step controllable from each time.

\section{Proof:}

Follows immediately from [11] and Definition 1.

\section{Definition 2.}

A VDD system that satisfies the conditions in Definition 1 is called $j$-step uncontrollable/unreachable over the interval $\left[i_{s}, i_{f}\right]$.

Definition 3 (Dual of Definition 1).

A VVD system is called $k$-step unobservable over the interval $\left[i_{s}-k, i_{f}\right] / k$-step unreconstructable over the interval $\left[i_{s}, i_{f}+k\right], \quad i_{0}+k \leq i_{s}<i_{f} \leq i_{N}-k$ if $\forall i \in\left[i_{s}-k, i_{f}\right]$ the system is not k-step observable at time $i$ / not k-step reconstructable from time $i+k$. 
Lemma 2 (dual of Lemma 1).

If $i_{s}, i_{f}$ satisfy the conditions in Definition 3 then over the interval $\left[i_{s}, i_{f}\right]$ the VDD system is 1) not k-step observable at each time and 2) not k-step reconstructable from each time.

Definition 4 (dual of Definition 2).

A VDD system that satisfies the conditions in Definition 3 is called $k$-step unreconstructable/unobservable over the interval $\left[i_{s}, i_{f}\right]$.

Application of the j-step k-step Kalman decomposition [11], [19] at each time $i \in\left[i_{0}, i_{N}\right]$, reveals all closed intervals (i.e. consisting of at least two consecutive discrete-time instants) where the system is $\mathrm{j}$-step uncontrollable/unreachable and dually all closed intervals where the system is $\mathrm{k}$-step unreconstructable/unobservable. As in Definition 2 and Definition 4 such intervals will be denoted by $\left[i_{s}, i_{f}\right]$. These closed intervals are precisely the intervals where stability of the closed loop system may be lost temporarily when designing static state and dynamic output feedback controllers.

Stabilizability is a property that relates entirely to the uncontrollable part of a system. A general approach to determine stabilizability is to extract this uncontrollable part, that is autonomous, by means of a Kalman decomposition, and to determine its stability. It will become clear in this section that application of a state basis transformation changes temporal stability and stabilizability properties. To recover them we therefore need to transform back to the original state basis. As opposed to this general approach, the stabilizability analysis presented in this section is much more straightforward and simple. It does not require transformation of the state basis because it relies fully on well established standard LQ theory applied to the original system representation. Therefore the associated numerical computations are also very efficient.

The stabilizability analysis in this section is unconventional in the sense that stability, stabilizability and detectability over finite time intervals is required. Stability over an interval relates to growth of the magnitude of the state over this interval. Throughout this paper $\|\bullet\|$ denotes the matrix 2 norm. For vectors this amounts to the L2 norm. In the next section we will demonstrate how to compute numerically the temporal and one-step stabilizability and detectability measures presented in this section, using only evaluations of the system matrices.

\section{Definition 5.}

An autonomous VVD system is called temporal stable over the interval $\left[i_{s}, i_{f}\right]$ if for any $x_{i_{s}} \neq 0,\left\|x_{i_{f}}\right\| /\left\|x_{i_{s}}\right\|<1$. 
Loosely speaking, according to Definition 5 an autonomous VDD system is called temporal stable over $\left[i_{s}, i_{f}\right]$ if for any initial state the magnitude of the associated terminal state is smaller than that of the initial state. An important difference between our definition and other finite-time stability concepts [13], [14] is that ours does not impose any restrictions on the magnitude of the state inside the interval. The advantage of Definition 5 is that it matches LQ control design as opposed to finitetime stability that relates to control system design using matrix inequalities [13] that is generally much more complicated.

\section{Definition 6.}

Associate to Definition 5 the following temporal stability measure,

$$
\rho\left(i_{s}, i_{f}\right)=\max _{x_{i_{s}} \neq 0}\left(\frac{\left\|x_{i_{f}}\right\|^{2}}{\left\|x_{i_{s}}\right\|^{2}}\right) \geq 0 .
$$

Observe that $\rho\left(i_{s}, i_{f}\right)$ in Definition 6 is the largest possible ratio $\left\|x_{i_{f}}\right\|^{2} /\left\|x_{i_{s}}\right\|^{2}$. This ratio matches the largest possible ratio $\left\|x_{i_{f}}\right\| /\left\|x_{i_{s}}\right\|$ in Definition 5. Therefore $\rho\left(i_{s}, i_{f}\right)$ is indeed a measure of temporal stability associated to Definition 5 . The smaller $\rho\left(i_{s}, i_{f}\right)$ the larger temporal stability. It will become clear that the squares in equation (1) are needed to achieve compatibility with LQ control computations.

\section{Theorem 1.}

An autonomous VDD system is temporal stable over the time interval $\left[i_{s}, i_{f}\right]$ if and only if,

$$
\rho\left(i_{s}, i_{f}\right)=\left\|\Phi_{i_{s}, i_{f}}^{T} \Phi_{i_{s}, i_{f}}\right\|<1
$$

where $\Phi_{i_{s}, i_{f}}$ represents the state transition matrix of the associated autonomous system from time $i_{s}$ to $i_{f}$.

\section{Proof:}

Because Theorem 1 applies to autonomous systems,

$$
x_{i_{f}}=\Phi_{i_{s}, i_{f}} x_{i_{s}} .
$$

Using equation (3) the temporal stability measure (1) becomes, 


$$
\begin{aligned}
& \rho\left(i_{s}, i_{f}\right)=\max _{x_{i_{s}} \neq 0}\left(\frac{\left\|\Phi_{i_{s}, i_{f}} x_{i_{s}}\right\|^{2}}{\left\|x_{i_{s}}\right\|^{2}}\right)= \\
& \max _{x_{i_{s}} \neq 0}\left(\frac{x_{i_{s}}^{T} \Phi_{i_{s}, i_{f}}^{T} \Phi_{i_{s}, i_{f}} x_{i_{s}}}{x_{i_{s}}^{T} x_{i_{s}}}\right)=\left\|\Phi_{i_{s}, i_{f}}^{T} \Phi_{i_{s}, i_{f}}\right\| .
\end{aligned}
$$

The last equality in equation (4) holds because $\Phi_{i_{s}, i_{f}}^{T} \Phi_{i_{s}, i_{f}}$ is nonnegative symmetric. Theorem 1 now follows from (4), Definition 5 and Definition 6 and,

$$
\left\|x_{i_{f}}\right\| /\left\|x_{i_{s}}\right\|<1 \Leftrightarrow\left\|x_{i_{f}}\right\|^{2} /\left\|x_{i_{s}}\right\|^{2}<1 .
$$

Stabilizability over a finite time-interval relates to the ability to stabilize the system over that interval by means of control.

\section{Definition 7.}

Associate to Definition 5 and Definition 6 the following temporal stabilizability measure that applies to VDD systems considered over the interval $i_{s}, i_{f}$,

$$
\rho_{\min }\left(i_{s}, i_{f}\right)=\max _{x_{i_{s}} \neq 0}\left(\frac{\min _{u_{i} \mid x_{i_{s}}}\left\|x_{i_{f}}\right\|^{2}}{\left\|x_{i_{s}}\right\|^{2}}\right) \geq 0,
$$

where $u_{i} \mid x_{i_{s}}$ indicates a control law dependent on $x_{i_{s}}$.

\section{Definition 8.}

A VDD system is called temporal stabilizable over $\left[i_{s}, i_{f}\right]$ if $\rho_{\min }\left(i_{s}, i_{f}\right)<1$.

\section{Theorem 2.}

A VDD system is temporal controllable over $\left[i_{s}, i_{f}\right] \Rightarrow \rho_{\min }\left(i_{s}, i_{f}\right)=0 \Rightarrow$ the VDD system is temporal stabilizable over $\left[i_{s}, i_{f}\right]$.

\section{Proof:}

If a VDD system is temporal controllable over $\left[i_{s}, i_{f}\right]$, then according to Definition 1 and [11], any state $x_{i_{s}}$ can be controlled to $x_{i_{f}}=0$. This implies $\rho_{\min }\left(i_{s}, i_{f}\right)=0$ and, according to Definition 8 , temporal stabilizability over $\left[i_{s}, i_{f}\right]$. 


\section{Remark 1.}

As with ordinary controllability and stabilizability, temporal controllability is a stronger property than temporal stabilizability.

To state the main theorem in this section consider the following parameterized discrete-time LQ problem. Given the system,

$$
x_{i+1}=\Phi_{i} x_{i}+\Gamma_{i} u_{i}, i \in\left[i_{s}, i_{f}-1\right] \text {, }
$$

with initial state,

$$
x_{i_{s}}
$$

find the control $u_{i}, i \in\left[i_{s}, i_{f}-1\right]$ that minimizes the cost function,

$$
J_{L Q}(\varepsilon)=x_{i_{f}}^{T} H x_{i_{f}}+\sum_{i=i_{s}}^{i_{f}-1}\left[x_{i}^{T} Q_{i} x_{i}+u_{i}^{T} R_{i}^{\varepsilon} u_{i}\right]
$$

with,

$$
H=I_{n}, Q_{i}=0, R_{i}^{\varepsilon}=\varepsilon I_{m}, 0 \leq \varepsilon \ll 1
$$

If $\varepsilon>0$ the Linear Quadratic control problem (7), (8)-(10) satisfies $H \geq 0, Q_{i} \geq 0, R_{i}^{\varepsilon}>0$. In this standard case it is well known that the optimal control is given by,

$$
u_{i}=-L_{i}^{\varepsilon} x_{i}, L_{i}^{\varepsilon}=\left(\Gamma_{i}^{T} S_{i+1}^{\varepsilon} \Gamma_{i}+R_{i}^{\varepsilon}\right)^{-1} \Gamma_{i}^{T} S_{i+1}^{\varepsilon} \Phi_{i},
$$

and the minimum cost by,

$$
J_{L Q}^{*}(\varepsilon)=x_{i_{s}}^{T} S_{i_{s}}^{\varepsilon} x_{i_{s}}
$$

where $S_{i}^{\varepsilon}, i \in\left[i_{s}, i_{f}\right]$ is the solution of the matrix Riccati difference equation,

$$
S_{i}^{\varepsilon}=\Phi_{i}^{T} S_{i+1}^{\mathcal{E}} \Phi_{i}-L_{i}^{T}\left(\Gamma_{i}^{T} S_{i+1}^{\mathcal{E}} \Gamma_{i}+R_{i}^{\varepsilon}\right) L_{i}+Q_{i}, S_{i_{f}}^{\mathcal{E}}=H
$$

\section{Theorem 3.}

$$
S_{i}^{*}=\lim _{\varepsilon \downarrow 0} S_{i}^{\varepsilon}
$$

exists, where $S_{i}^{\varepsilon}, \mathcal{E} \downarrow 0$ satisfies the matrix Riccati difference equation (13) with data as specified by equation (10). Furthermore, 


$$
\rho_{\min }\left(i, i_{f}\right)=\left\|S_{i}^{*}\right\|, i \in\left[i_{s}, i_{f}-1\right]
$$

As a special case of (15),

$$
\rho_{\min }\left(i_{s}, i_{f}\right)=\left\|S_{i_{s}}^{*}\right\|
$$

\section{Proof:}

First observe that in the parameterized LQ problem (7)-(10) we may replace the initial time $i_{s}$ by $i^{\prime} \in\left[i_{s}, i_{f}-1\right]$. This also hold for the stabilizability measure $\rho_{\min }$. Next from equations (9), (10) observe that

$$
\min _{u_{i} \mid x_{i^{\prime}}} J_{L Q}(0)=\min _{u_{i} \mid x_{i^{\prime}}}\left(x_{i_{f}}^{T} x_{i_{f}}\right)=\min _{u_{i} \mid x_{i^{\prime}}}\left\|x_{i_{f}}\right\|^{2}
$$

Now the key to proving (14), (15) is to prove that,

$$
\min _{u_{i} \mid x_{i^{\prime}}} J_{L Q}(0)=\lim _{\varepsilon \downarrow 0} J_{L Q}^{*}(\varepsilon)=x_{i^{\prime}}^{T} S_{i^{\prime}}^{*} x_{i^{\prime}}
$$

Suppose equation (18) holds. Then from equations (6), (17), (18),

$$
\begin{aligned}
& \rho_{\min }\left(i^{\prime}, i_{f}\right)=\max _{x_{i^{\prime}} \neq 0}\left(\frac{\min _{u_{i} \mid x_{i^{\prime}}}\left\|x_{i_{f}}\right\|^{2}}{\left\|x_{i^{\prime}}\right\|^{2}}\right)= \\
& \max _{x_{i^{\prime}}}\left(\frac{x_{i^{\prime}}^{T} S_{i^{\prime}}^{*} x_{i^{\prime}}}{x_{i^{\prime}}^{T} x_{i^{\prime}}}\right)=\left\|S_{i^{\prime}}^{*}\right\|
\end{aligned}
$$

The last equality in equation (19) holds because $S_{i^{\prime}}^{*}$ is nonnegative symmetric. So we are left to prove equation (18). Consider the j-step, k-step Kalman decomposition at time $i_{f}$ with $j=i_{f}-i^{\prime}$. According to this decomposition the linear system (7) can be decomposed into a part that is j-step controllable from time $i^{\prime}$ and a part that is autonomous. The contribution of the j-step controllable part to $\min _{u_{i} \mid x_{i^{\prime}}} J_{L Q}(0)$ is zero. The contribution to $J_{L Q}^{*}(\varepsilon)$ tends to zero as $\varepsilon \downarrow 0$. The contribution of the autonomous part to both $\min _{u_{i} \mid x_{i}} J_{L Q}(0)$ and $J_{L Q}^{*}(\varepsilon)$ is fixed and independent of $\varepsilon$. Because the system matrices are bounded this contribution is also finite. This proves the existence of the limit (16) and the equality (18). 


\section{Remark 2.}

There are three reasons for considering $0<\varepsilon \ll 1$ in equation (10), instead of $\mathcal{E}=0$. Taking $0<\varepsilon \ll 1, \varepsilon$ may be used to 1) keep the control within certain bounds that apply in practice and 2) as a numerical tolerance to prevent illconditioning of the computation of equation (11) when $\Gamma_{i}^{T} S_{i+1}^{\mathcal{E}} \Gamma_{i}$ is not full rank and $L_{i} \rightarrow \infty$ as $\varepsilon \downarrow 0$. In practice the selection of $0<\varepsilon \ll 1$ will be a compromise and $S_{i}^{\varepsilon}$ will approximate $S_{i}^{*}, i \in\left[i_{s}, i_{f}-1\right]$. As a result all computations in this paper involving $S_{i}^{\varepsilon}$ will be approximations, although generally very good ones. Thirdly $\varepsilon=0$ leads to a singular LQ problem that is generally much more difficult to solve and the solution of which need not be unique.

When analyzing control systems the state behavior over the entire interval $\left[i_{s}, i_{f}\right]$ is generally of interest, not just the behavior at the initial time $i_{s}$ and the final time $i_{f}$. This behavior is partly considered by equation (15) of Theorem 3 that determines the stabilizability measure for each sub interval $\left[i, i_{f}\right], i \in\left[i_{s}, i_{f}-1\right]$. The following theorem introduces a one-step stabilizability measure that applies to individual time instants.

\section{Theorem 4.}

$\left\|S_{i}^{*}\right\|-\left\|S_{i+1}^{*}\right\|$ is a one-step stabilizability measure (os-stabilizability measure) at time $i \in\left[i_{s}, i_{f}-1\right]$.

\section{Proof:}

From (15),

$$
\rho_{\min }\left(i_{s}, i_{f}\right)=\left\|S_{i_{s}}^{*}\right\|=\left\|S_{i_{f}}^{*}\right\|+\sum_{i=i_{s}}^{i_{f}-1}\left(\left\|S_{i}^{*}\right\|-\left\|S_{i+1}^{*}\right\|\right)
$$

so $\left\|S_{i}^{*}\right\|-\left\|S_{i+1}^{*}\right\|, \quad i \in\left[i_{s}, i_{f}-1\right]$ is the one-step contribution at time $i$ to the temporal stabilizability measure $\rho_{\min }\left(i_{s}, i_{f}\right)$. If this contribution is negative $\rho_{\min }\left(i_{s}, i_{f}\right)$ decreases and temporal stabilizability increases.

\section{Definition 9.}

A VDD system is called one-step stabilizable (os-stabilizable) at time $i \in\left[i_{s}, i_{f}-1\right]$ if $\left\|S_{i}^{*}\right\|-\left\|S_{i+1}^{*}\right\|<0$. 
Because for a VDD system temporal and one-step detectability are dual to temporal and one-step stabilizability, the following definitions and theorems are stated without further explanation and proof.

Theorem 5 (dual of Theorem 3).

$$
P_{i}^{*}=\lim _{\varepsilon \downarrow 0} P_{i}^{\varepsilon}
$$

exists, where $P_{i}^{\varepsilon}, \mathcal{E} \downarrow 0$ satisfies the matrix Riccati difference equation that is dual to $(13)$,

$$
P_{i+1}^{\varepsilon}=\Phi_{i} P_{i}^{\varepsilon} \Phi_{i}^{T}-L_{i}^{\varepsilon}\left(C_{i} P_{i}^{\varepsilon} C_{i}^{T}+R_{i}\right) L_{i}^{\varepsilon T}+Q_{i}, P_{i_{s}}^{\varepsilon}=H
$$

with,

$$
L_{i}^{\varepsilon}=\Phi_{i} P_{i}^{\varepsilon} C_{i}^{T}\left(C_{i} P_{i}^{\varepsilon} C_{i}^{T}+R_{i}^{\varepsilon}\right)^{-1}
$$

with data as specified by equation (10). Furthermore,

$$
\sigma_{\min }\left(i_{s}, i\right)=\left\|P_{i}^{*}\right\|, i \in\left[i_{s}+1, i_{f}\right]
$$

where $\sigma_{\min }\left(i, i_{f}\right)$ is a temporal detectability measure over the interval $\left[i, i_{f}\right]$. As a special case,

$$
\sigma_{\min }\left(i_{s}, i_{f}\right)=\left\|P_{i_{f}}^{*}\right\|
$$

Definition 10 (dual of Definition 8).

A VDD system is called temporal detectable over $\left[i_{s}, i_{f}\right]$ if $\sigma_{\min }\left(i_{s}, i_{f}\right)<1$.

Theorem 6 (dual of Theorem 4).

$\left\|P_{i+1}^{*}\right\|-\left\|P_{i}^{*}\right\|$ is a one-step detectability measure (os-detectability measure) at time $i \in\left[i_{s}, i_{f}-1\right]$.

Definition 11 (dual of Definition 9).

A VDD system is called one-step detectable (os-detectable) at time $i \in\left[i_{s}, i_{f}-1\right]$ if $\left\|P_{i+1}^{*}\right\|-\left\|P_{i}^{*}\right\|<0$. 


\section{Conclusions}

New temporal properties and associated measures for control system design concerning time-varying linear discrete-time systems were introduced in this paper. The properties and associated measures concern temporal and one-step stabilizability and detectability. They indicate to what extent control system design is problematic when discrete-time linear time-varying systems are temporal uncontrollable or temporal unreconstructable. Temporal uncontrollability and unreconstructability are detected by the j-step, k-step Kalman decomposition. As demonstrated in this paper, after introduction of a suitable, simple stability property, that applies over finite time intervals, application of ordinary standard LQ theory and algorithms enables the computation of associated temporal and one-step stabilizability and detectability measures. These determine to what extent a static or dynamic feedback control system becomes temporal unstable. A major application concerns the temporal stability analysis of digital perturbation output feedback controllers for nonlinear systems tracking control and state trajectories that may be optimal [1], [10].

As an alternative to LQ theory, temporal stabilizability may be determined by extracting the temporal uncontrollable or temporal unreconstructable subsystems and analyzing their temporal stability. In principle, the j-step, k-step Kalman decomposition is able to extract these subsystems. The extraction employs state basis transformations that generally change temporal stability properties. The approach presented in this paper is more simple and direct because it applies standard LQ theory to the original, untransformed system.

Although the LQ problems in this paper are singular in principle, it is advantageous to approximate them by non-singular LQ problems, as demonstrated in this paper. The interpretation of $\left\|S_{i}^{*}\right\|$ as a temporal stabilizability measure is new and highly interesting. The same applies to the interpretation of $\left\|S_{i}^{*}\right\|-\left\|S_{i+1}^{*}\right\|$ as a one-step stabilizability measure that measures the contribution to stabilizability of each single time-step.

Along the lines of this paper we are also currently exploring temporal properties of time-varying linear systems with white stochastic parameters [20]. Among others these enable robust digital optimal perturbation feedback design for nonlinear systems.

\section{References}

[1] Athans, M.: The role and use of the Linear- Quadratic-Gaussian problem in control system design. IEEE Trans. Aut. Contr. 16, 529-552 (1971)

[2] van Willigenburg, L.G., De Koning, W.L.: On the synthesis of time-varying LQG weights and noises along optimal control and state trajectories. Optimal Control Applications and Methods 27, 137-160 (2006) 
[3] Van Willigenburg, L.G., De Koning, W.L.: A Kalman decomposition to detect temporal linear system structure. In: Proceedings European Control Conference, Kos, Greece, July 2-7, Paper nr. 78, 6 p. (2007)

[4] Van Willigenburg, L.G., De Koning, W.L.: Temporal linear system structure. IEEE Trans. Aut. Contr. 53(5), 1318-1323 (2008)

[5] Van Willigenburg, L.G., De Koning, W.L.: Temporal and differential stabilizability and detectability of piecewise constant rank systems. Optimal Control Application \& Methods, published online in Wiley Online Library (wileyonline library.com) (2011), doi:10.1002/oca.997

[6] Jameson, A., O'Malley, R.E.: Cheap control of the time-invariant regulator. Appl. Math. \& Optimization 1(4), 337-354 (1975)

[7] Kokotovic, P.V., O'Malley, R.E., Sannuti, P.: Singular perturbations and order-reduction in control theory - an overview. Automatica 12, 123-132 (1976)

[8] Levis, A.H., Schlueter, R.A., Athans, M.: On the behavior of optimal linear sampled-data regulators. International Journal of Control 13, 343-361 (1971)

[9] Van Willigenburg, L.G., De Koning, W.L.: The digital optimal regulator and tracker for stochastic time-varying systems. International Journal of Systems Science 12, 2309-2322 (1992)

[10] Van Willigenburg, L.G.: Digital optimal control and LQG compensation of asynchronous and aperiodically sampled nonlinear systems. In: Proceedings 3rd European Control Conference, Rome, Italy, vol. 1, pp. 496-500 (September 1995)

[11] van Willigenburg, L.G., De Koning, W.L.: Temporal linear system structure: The discrete-time case. In: Proceedings of the ECC 2009, Budapest, August 23-26, pp. 225-230 (2009)

[12] Kalman, R.E., Bertram, J.E.: Control system design via the "Second Method" of Lyapunov, I Continuous-time systems. Transactions of the ASME, Journal of Basic Engineering, 371-393 (June 1960)

[13] Amato, F., Ariola, M., Carbone, M., Cosentiono, C.: Finite-Time Control of Linear Systems: A Survey. In: Current Trends in Nonlinear Systems and Control. Systems and Control: Foundations \& Applications, Part II, pp. 195-213 (2006)

[14] Amato, F., Ambrosino, R., Ariola, M., Cosentino, C.: Finite-time stability of linear timevarying systems with jumps. Automatica 45, 1354-1358 (2009)

[15] Gohberg, I., Kaashoek, M.A., Lerer, L.: Minimality and realization of discrete timevarying systems. Operator Theory: Advances and Applications 56, 261-296 (1992)

[16] Van Willigenburg, L.G., De Koning, W.L.: Minimal and non-minimal optimal fixedorder compensators for time-varying discrete-time systems. Automatica 38, 157-165 (2002)

[17] Sandberg, H., Rantzer, A.: Balanced truncation of linear time-varying systems. IEEE Trans. Aut. Contr. 49(2), 217-229 (2004)

[18] Van Willigenburg, L.G., De Koning, W.L.: Linear systems theory revisited. Automatica 44, 1686-1696 (2008)

[19] Boley, D.: Computing the Kalman decomposition: An optimal method. IEEE Trans. Aut. Contr. 29(1), 51-53 (1984)

[20] van Willigenburg, L.G., De Koning, W.L.: Compensatability and optimal compensation of systems with white parameters in the delta domain. International Journal of Control 83(12), 2546-2563 (2010) 